\title{
Development and implementation of treadmill exercise testing protocols in COPD
}

This article was published in the following Dove Press journal: International Journal of Chronic Obstructive Pulmonary Disease II October 2010

Number of times this article has been viewed

\section{Christopher B Cooper' Marlon Abrazado' \\ Daniel Legg ${ }^{2}$ \\ Steven Kesten ${ }^{2}$ \\ 'David Geffen School of Medicine, University of California, Los Angeles, CA, USA; ${ }^{2}$ Boehringer Ingelheim Pharmaceuticals Inc., Ingelheim, Germany}

Background: Because treadmill exercise testing is more representative of daily activity than cycle testing, we developed treadmill protocols to be used in various clinical settings as part of a two-year, multicenter, chronic obstructive pulmonary disease (COPD) trial evaluating the effect of tiotropium on exercise.

Methods: We enrolled 519 COPD patients aged 64.6 \pm 8.3 years with a postbronchodilator forced expiratory volume in one second $\left(\mathrm{FEV}_{1}\right)$ of $1.25 \pm 0.42 \mathrm{~L}, 44.3 \% \pm 11.9 \%$ predicted. The patients performed symptom-limited treadmill tests where work rate $(\dot{W})$ was increased linearly using speed and grade adjustments every minute. On two subsequent visits, they performed constant $\dot{W}$ tests to exhaustion at $90 \%$ of maximum $\dot{W}$ from the incremental test.

Results: Mean incremental test duration was $522 \pm 172$ seconds (range 20-890), maximum work rate $66 \pm 34$ watts. For the first and second constant $\dot{W}$ tests, both at $61 \pm 33$ watts, mean endurance times were $317 \pm 61$ seconds and $341 \pm 184$ seconds, respectively. The mean of two tests had an intraclass correlation coefficient of $0.85(P<0.001)$. During the second constant $\dot{W}$ test, $88.2 \%$ of subjects stopped exercise because of breathing discomfort; $87.1 \%$ for Global Initiative for Chronic Obstructive Lung Disease (GOLD) Stage II, 88.5\% for GOLD Stage III, and $90.2 \%$ for GOLD Stage IV.

Conclusion: The symptom-limited incremental and constant work treadmill protocol was well tolerated and appeared to be representative of the physiologic limitations of COPD.

Keywords: chronic obstructive pulmonary disease, exercise testing, endurance, tiotropium

\section{Introduction}

Patients with chronic obstructive pulmonary disease (COPD) have limited exercise capacity due to complex pathophysiology, and evaluation of exercise performance at all stages of COPD is important if we are to understand disease progression better. ${ }^{1}$ One approach would be to measure aerobic capacity ( $\mathrm{VO}_{2} \max$ ) periodically. However, there has been limited development of maximal incremental protocols specific for patients with COPD. Furthermore, maximal incremental exercise testing is effort-dependent and not representative of everyday activity. Functional exercise tests, such as six-minute walking distance, vary significantly in how they are performed from center to center, despite guidelines. ${ }^{2,3}$ Six-minute walking distance may not reflect maximal ability, because it is self-paced and also may not be sensitive to pharmacologic intervention. ${ }^{3,4}$ Oga et $\mathrm{al}^{5}$ reported that among the different types of exercise test, the submaximal constant work rate endurance test was the most sensitive in detecting improvements after bronchodilator therapy. Other investigators have successfully used this type of test to elucidate physiologic impairments in COPD. ${ }^{6,7}$
Correspondence: Christopher B Cooper David Geffen School of Medicine, University of California, Los Angeles, I0833 Le Conte Avenue, 37-I3 I CHS, Los Angeles, CA 90095-1690, USA

Tel + I 3108254440

Fax + I 310206821 I

Email ccooper@mednet.ucla.edu 
A similar type of test, the endurance shuttle walk, was tested at approximately $75 \%, 85 \%$, and $95 \%$ of maximum intensity and found to be both repeatable and sensitive to therapeutic changes. ${ }^{8}$

Cycle ergometry testing has traditionally been used to assess the efficacy of treatments. However, walking is less likely to induce quadriceps fatigue and is more often limited by breathlessness compared with cycling, ${ }^{9}$ and therefore may better reflect the impact of pharmacologically induced changes in lung function on exercise tolerance. ${ }^{10,11}$ Computer control enables electronically braked cycle exercise to be implemented with a continuous linear increase in work rate, but now linear treadmill protocols have also been described. ${ }^{12}$ Furthermore, it has been shown that treadmill versus cycle exercise elicits higher levels of ventilation and is more likely to be associated with oxyhemoglobin desaturation. ${ }^{13}$ Thus, exercise testing using a treadmill may better reflect the activities of daily living than cycle ergometry.

In order to overcome some of the problems associated with cycle ergometry, we developed symptom-limited, incremental, and constant work rate $(\dot{W})$ protocols using a treadmill to measure exercise performance in patients with COPD. The maximal incremental treadmill protocol was designed with an approximate linear increase in work rate with respect to time. We then implemented duplicate constant load exercise tests at $80 \%$ and $90 \%$ of the maximum work rate achieved in the preceding incremental test. The repeatability of these constant load exercise tests was evaluated in a small pilot study. The objective was to standardize a treadmill protocol that would be acceptable to COPD patients with a wide range of disease severity and would be suitable for use in diverse clinical settings, including an international, multicenter, interventional clinical trial called EXACTT (a randomized, double-blind, placebo-controlled two-year trial to examine the changes in exercise endurance and COPD treated with tiotropium once daily).

Tiotropium has been documented to improve constant work cycle exercise duration in two six-week trials and to improve constant speed treadmill exercise in a six-month trial in a pulmonary rehabilitation setting. ${ }^{6,7,14}$ With the EXACTT trial, we are seeking to evaluate whether improvements in exercise duration with tiotropium in a relatively broad selection of COPD patients could be sustained over two years using a COPD-specific novel constant work treadmill exercise protocol.

\section{Methods}

\section{Exercise protocol development}

The maximal incremental treadmill exercise protocol was developed by increasing the work rate at one-minute intervals, with the target being symptom limitation in approximately 8-12 minutes..$^{15}$ In order to achieve a near-linear increase in work rate, a calculated sequence of speed and grade adjustments was developed from basic principles. ${ }^{16,17} \mathrm{We}$ incorporated a more gradual work rate incrementation early in the test specifically designed for impaired subjects. The speed and grade adjustments, shown in Table 1, were standardized across subjects to maintain the linearity of the incremental protocol, but the actual work rates varied according to body weight. The work rates shown as an example in Table 1 assume a subject weighing $70 \mathrm{~kg}$.

A small feasibility study was performed with 12 COPD patients at the UCLA Exercise Physiology Research Laboratory. Each subject attended at the same time of day on three occasions. On the first visit, they performed an incremental treadmill test using the adjustments of speed and grade as shown in Table 1. On two subsequent visits, they performed constant $\dot{W}$ tests to exhaustion either at $80 \%$

Table I Speed and grade adjustments used in incremental treadmill exercise

\begin{tabular}{lllll}
\hline $\begin{array}{l}\text { Time } \\
(\mathbf{m i n}) *\end{array}$ & $\begin{array}{l}\text { Speed } \\
(\mathbf{m p h})\end{array}$ & $\begin{array}{l}\text { Speed } \\
(\mathbf{m} / \mathbf{s e c})\end{array}$ & $\begin{array}{l}\text { Grade } \\
(\%)\end{array}$ & $\begin{array}{l}\text { Work } \\
\text { (watts)** }\end{array}$ \\
\hline WI & $\mathrm{I}$ & 0.45 & 0 & "0" \\
W2 & $\mathrm{I}$ & 0.45 & 0 & "0" \\
W3 & $\mathrm{I}$ & 0.45 & 0 & "0" \\
E0 & $\mathrm{I}$ & 0.45 & $\mathrm{I}$ & 3 \\
EI & $\mathrm{I}$ & 0.45 & 2 & 6 \\
E2 & $\mathrm{I}$ & 0.45 & 3 & 9 \\
E3 & $\mathrm{I}$ & 0.45 & 5 & 15 \\
E4 & 1.5 & 0.67 & 5 & 23 \\
E5 & 1.5 & 0.67 & 7 & 32 \\
E6 & 2 & 0.89 & 7 & 43 \\
E7 & 2 & 0.89 & 8 & 49 \\
E8 & 2.5 & 1.12 & 8 & 61 \\
E9 & 2.5 & 1.12 & 9 & 69 \\
EI0 & 3 & 1.34 & 9 & 83 \\
EII & 3.5 & 1.56 & 9 & 96 \\
EI2 & 4 & 1.79 & 9 & 110 \\
EI3 & 4.5 & 2.01 & 9 & 124 \\
EI4 & 5 & 2.24 & 9 & 138 \\
RI & $I$ & 0.45 & 0 & "0" \\
R2 & I & 0.45 & 0 & "0"
\end{tabular}

Notes: *WI-W3 represents a three-minute warm-up period. E0-EI4 represents a I5-minute incremental exercise phase. RI-R2 represents a two-minute cool-down period; **Work rate is calculated based on treadmill speed and grade for a subject weighing $70 \mathrm{~kg}$. "0" is notionally "zero watts" for the warm-up and cool-down phases. 
or $90 \%$ of maximum $\dot{W}$ determined from the incremental test. During each test, minute ventilation $\left(\dot{V}_{E}\right)$ and heart rate $\left(f_{c}\right)$ were continuously monitored. Oxygen uptake $\left(\dot{V} O_{2}\right)$ and carbon dioxide output $\left(\dot{\mathrm{V} C \mathrm{O}_{2}}\right)$ were derived from exhaled gas analysis using a metabolic measurement system (Vmax; VIASYS Healthcare, Yorba Linda, CA). This system was calibrated using standard routines prior to each exercise test. Subjects received standard encouragement during the tests using a novel instrument (Figure 1). At the end of the tests, rating of perceived exertion was obtained using the original Borg RPE scale, ${ }^{18}$ and breathlessness was evaluated using a $100 \mathrm{~mm}$ visual analog scale.

For the purpose of endurance testing, a fixed percentage of the maximum work rate was applied as a constant work rate, and time to exhaustion was measured as the outcome of interest. The standard approach to constant work rate cycle ergometry has been to initiate the test at $75 \%$ or $80 \%$ maximum work rate. ${ }^{6,7}$ In the feasibility study, we therefore used $80 \%$ as a starting point for constant work treadmill testing. However, it became apparent that $80 \%$ of maximal work during a treadmill test resulted in a prolonged exercise time beyond the target range in a number of patients. Hence the constant work was reset to $90 \%$ of maximum work. The chosen constant work rate (eg, $80 \%$ or $90 \%$ of maximum) is easily derived by proportional reduction of the final speed from the incremental test, leaving the final grade unchanged. For example, if one desired $90 \%$ of maximum work rate in a subject whose maximal incremental treadmill settings were a speed of $1.34 \mathrm{~m} / \mathrm{sec}(3 \mathrm{mph})$ and grade of $9 \%$, then the appropriate settings would be a speed of $1.21 \mathrm{~m} / \mathrm{sec}(2.7 \mathrm{mph})$, ie, $90 \%$ of maximum speed, and the same grade of $9 \%$.

\section{EXACTT protocol}

The maximal incremental and 90\% constant work rate treadmill exercise testing protocols described above were incorporated into the design of the EXACTT study, which aimed to examine the effects of tiotropium on exercise endurance in COPD patients over two years. The goal of EXACTT was to recruit over 500 subjects and this has been achieved using 61 sites in 11 different countries. EXACTT included men and women, aged 40-80 years, with a clinical diagnosis of COPD, ratio of the forced expiratory volume in

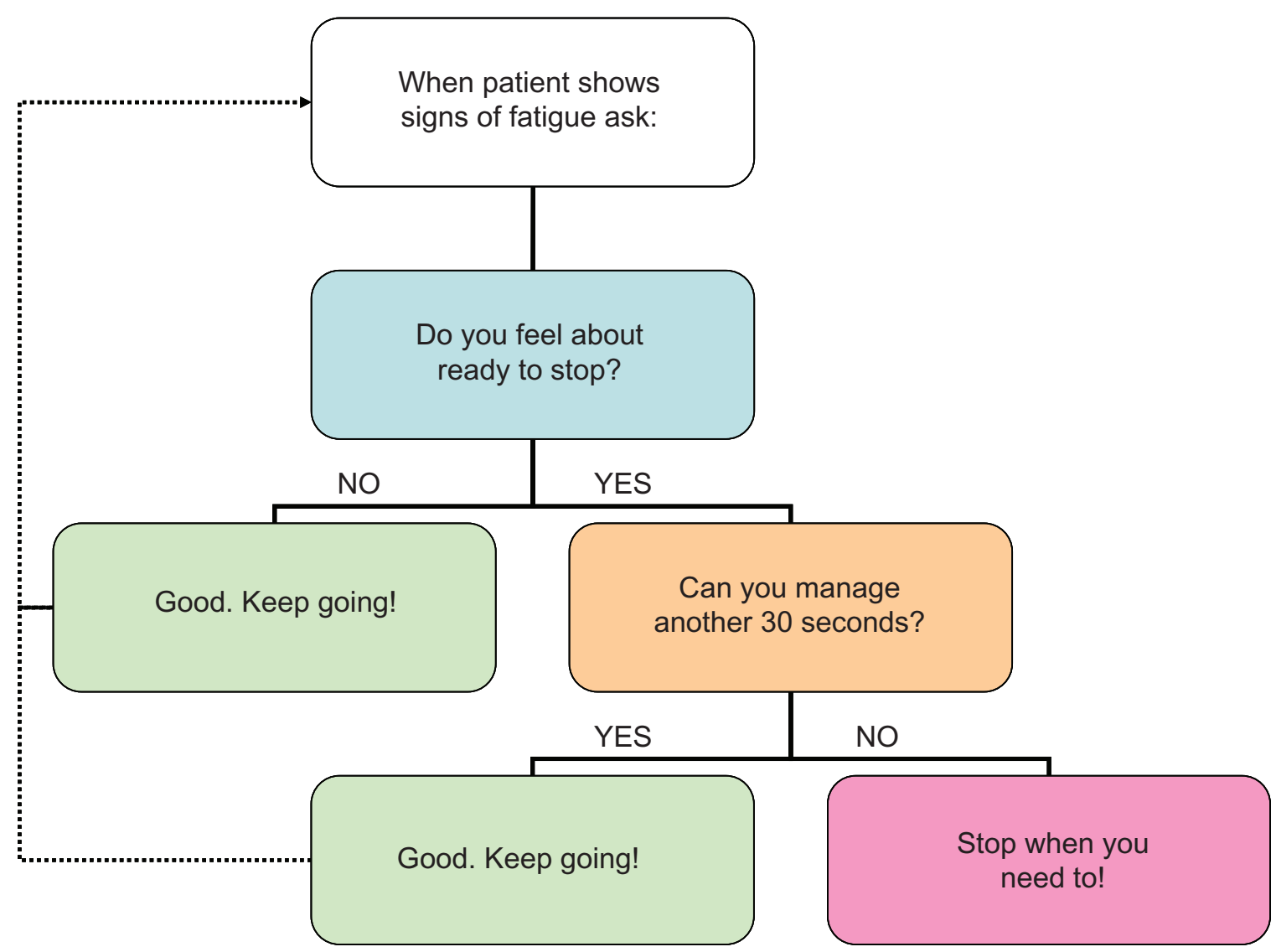

Figure I EXACTT instrument used to optimize maximal effort during incremental and endurance exercise tests. 
the first one second to the forced vital capacity of the lungs $\left(\mathrm{FEV}_{1} / \mathrm{FVC}\right)<70 \%$ and a smoking history greater than 10 pack-years. Spirometry was performed in accordance with American Thoracic Society/European Respiratory Society standards. ${ }^{19,20}$ Measures of FEV 1 and FVC were compared with reference values from the Third National Health and Nutrition Examination Survey (NHANES III). ${ }^{21}$ Patients with pulmonary disease other than COPD, including asthma, clinically significant bronchiectasis, interstitial lung disease, pleural disease, and previous history of thoracic surgery, such as lung resection or lung volume reduction surgery, were excluded. Patients requiring supplemental oxygen at rest or during exercise to prevent desaturation $(<85 \%)$ were also excluded. Patients with disorders that could impact their ability to participate in exercise testing, such as obesity (body mass index $>30 \mathrm{~kg} / \mathrm{m}^{2}$ ), and cardiovascular or musculoskeletal disease were excluded. Patients were allowed to continue taking other prescribed medications, including long-acting $\beta$-sympathomimetic bronchodilators and inhaled corticosteroids, with the same timing and dosage throughout the study. The following respiratory medications were washed out prior to each clinic visit for exercise testing: short-acting theophylline ( 24 hours), long-acting theophylline (48 hours), inhaled corticosteroids (12 hours), long-acting inhaled beta-agonists ( 24 hours), combination inhaled corticosteroid-long-acting beta-agonists ( 24 hours), and short-acting beta-agonists (eight hours). At the beginning of the EXACTT study, subjects had treadmill exercise testing on three visits. At visit 1, they performed the incremental protocol. At visits 2 and 3, constant work rate protocols were performed at $90 \%$ of the initial maximum work rate. Visits 1,2 , and 3 were separated by seven or 14 days, depending on tiotropium use at consent as specified in the protocol. The exercise tests on visits 2 and 3 were separated by a minimum of three days. The only data captured from these tests were for endurance time. Gas exchange measurements were not included in the protocol for the EXACTT study. Subjects were permitted to use the rails for balance but were requested not to hold onto the rails. Standardization was achieved through a central training and an interactive DVD/web-based training system. Successful completion of training was required for all study staff conducting exercise testing. Treadmills were calibrated annually according to the procedures described in the National Aeronautics and Space Administration manual entitled "Procedures for Exercise Laboratories" (http://ston. jsc.nasa.gov/collections/TRS/_techrep/TM-1998-104826. pdf). After baseline testing, the subjects are being followed for two years with three-monthly measurements of constant work rate treadmill exercise endurance time at $90 \%$ of the initial maximum work rate. This paper presents data from the pilot feasibility study, as well as the baseline characteristics, before randomization, of the subjects recruited into the EXACTT study.

\section{Statistical analysis}

Data were analyzed using the SAS software package (version 9.2; SAS Institute, Cary, NC). The subject characteristics, including endurance times for the incremental tests (visit 1 ) and paired constant $\dot{W}$ tests (visits 2 and 3 ), are presented as mean values with standard deviations (SD). The reproducibility of exercise tests was assessed by computing intraclass correlation coefficient for the mean of two tests. ${ }^{22}$ Intraclass correlation scores are based on Shrout and Fleiss and present the two-way mixed average. ${ }^{23}$

Based on two previous tiotropium exercise trials using constant work rate cycle ergometry, the SD of exercise duration was assumed to be around 300 seconds. ${ }^{6,7}$ The difference between tiotropium and placebo for the constant work rate test was approximately 105 seconds. A total of 173 patients in each group would provide $90 \%$ power to detect a 105 -second difference in exercise duration at the 0.025 significance level. Given that the primary endpoint might not be normally distributed, a sample size of 184 in each group would be needed using a Wilcoxon (Mann-Whitney) rank-sum test. To ensure sufficient power for analyzing the primary endpoint in the set of patients treated for at least 48 weeks and assuming a discontinuation rate of about $20 \%$, the sample size was set at 230 patients per group.

\section{Results}

\section{Feasibility study}

For the feasibility study, we recruited 12 clinically stable COPD patients (nine men, three women), mean age $69.5 \pm 7.8$ years, $\mathrm{FEV}_{1} 1.44 \pm 0.38 \mathrm{~L}(33 \%-70 \%$ of reference). The important parameters of their exercise tests are summarized in Table 2. The mean incremental test duration approximated 10 minutes. Plots of $\dot{V} O_{2}$ versus time indicated linear increases in $\dot{V} \mathrm{O}_{2}$ during incremental testing, with a mean gradient of $\dot{V} \mathrm{O}_{2}$ versus $\dot{W}$ of $10.7 \pm 4.3 \mathrm{~mL} / \mathrm{min} /$ watt, indicating work efficiency similar to that of cycle ergometry. ${ }^{24}$ Seven patients demonstrated a $\dot{V} O_{2}$ plateau at the end of the test, confirming maximal effort. A metabolic threshold $\left(\dot{V} \mathrm{O}_{2} \theta\right)$, above which lactic acid was deduced to have accumulated, was identified in 11/12 subjects by noninvasive gas exchange measurements using the method of Beaver et $\mathrm{al}^{25}$ in conjunction with analysis of the ventilatory equivalents 
Table 2 Physiologic data from the pilot feasibility study

\begin{tabular}{|c|c|c|c|c|c|}
\hline \multirow[t]{2}{*}{ Parameter } & \multirow{2}{*}{$\begin{array}{l}\text { Maximal incremental } \\
\text { Visit I }\end{array}$} & \multicolumn{2}{|c|}{ Constant $80 \% \dot{W} \max$} & \multicolumn{2}{|c|}{ Constant $90 \% \dot{W} \max$} \\
\hline & & Visit 2 & Visit 3 & Visit 2 & Visit 3 \\
\hline Time (seconds) & $575(170)$ & $599(22 I)$ & $713(267)$ & $308(180)$ & $388(235)$ \\
\hline Speed (mph) & $2.7(1.0)$ & $2.2(0.7)$ & $2.2(0.7)$ & $2.6(0.8)$ & $2.6(0.8)$ \\
\hline Grade (\%) & $8.2(1.6)$ & $8.4(1.4)$ & $8.4(1.4)$ & $8.1(1.5)$ & $8.1(1.5)$ \\
\hline$\dot{W}$ (watts) & $80(38)$ & $67(29)$ & $68(30)$ & $77(34)$ & $77(34)$ \\
\hline $\mathrm{VO}_{2} \max (\mathrm{L} / \mathrm{min})$ & $\mathrm{I} .34(0.36)$ & $\mathrm{I} .28(0.35)$ & $1.26(0.37)$ & I.4I (0.39) & $\mathrm{I} .40(0.43)$ \\
\hline $\mathrm{VO}_{2} \max (\%$ reference) & $86(23)$ & $81(22)$ & $79(21)$ & $96(29)$ & $95(26)$ \\
\hline $\mathrm{VO}_{2} \max (\mathrm{mL} / \mathrm{kg} / \mathrm{min})$ & $16.7(4.2)$ & $15.2(2.9)$ & $14.9(2.9)$ & $17.6(4.3)$ & $17.3(3.9)$ \\
\hline$\dot{\mathrm{V}} \mathrm{O}_{2} / \mathrm{W}(\mathrm{mL} / \mathrm{min} / \mathrm{watt})$ & $10.7(4.3)$ & - & - & - & - \\
\hline$\dot{\mathrm{VO}}_{2} \theta(\mathrm{L} / \mathrm{min})$ & $0.98(0.17)$ & - & - & - & - \\
\hline$\dot{\mathrm{V}} \mathrm{O}_{2}\left(6^{\prime}-3^{\prime}\right)(\mathrm{L} / \mathrm{min})$ & - & $0.05(0.08)$ & $\mathrm{I} .22(0.4 \mathrm{I})$ & $0.13(0.03)$ & $0.12(0.08)$ \\
\hline$\dot{\mathrm{V} C \mathrm{O}_{2}} \max (\mathrm{L} / \mathrm{min})$ & $1.29(0.40)$ & $1.22(0.37)$ & $\mathrm{I} .22(0.4 \mathrm{I})$ & $\mathrm{I} .4 \mathrm{I}(0.5 \mathrm{I})$ & $\mathrm{I} .35(0.50)$ \\
\hline$f_{c} \max (/ \min )$ & $119(17)$ & $113(14)$ & $112(13)$ & $122(17)$ & $119(13)$ \\
\hline$f_{c} \max (\%$ reference) & $80(13)$ & $75(\mathrm{II})$ & $74(11)$ & $81(10)$ & $79(8)$ \\
\hline$\dot{V}_{E} \max (\mathrm{L} / \mathrm{min})$ & $43.7(11.6)$ & $44.2(13.2)$ & $45.1(15.4)$ & $46.8(14.6)$ & $48.4(17.4)$ \\
\hline$\dot{V}_{E} \max / M V V(\%)$ & $77(18)$ & $68(16)$ & $69(20)$ & $85(\mathrm{II})$ & $87(\mathrm{II})$ \\
\hline RPE (Borg 6-20) & $15.0(1.8)$ & - & - & $14.8(2.1)$ & I5.I (2.2) \\
\hline Dyspnea (VAS 0-100) & $50(20)$ & - & - & $52(28)$ & $58(28)$ \\
\hline
\end{tabular}

Note: Values are mean ( \pm standard deviation).

Abbreviations: $\dot{W}$, work rate; $\dot{V} \mathrm{O}_{2}$, oxygen uptake; $\dot{\mathrm{VO}}_{2}\left(6^{\prime}-3^{\prime}\right)$, change in oxygen uptake between the third and sixth minutes of the test (oxygen uptake drift); $\dot{V} \mathrm{CO} \mathrm{O}_{2}$, carbon dioxide output, $f_{c}$, heart rate; $\dot{V}_{E}$, minute ventilation; $M V V$, maximum voluntary ventilation; RPE, rating of perceived exertion (on original Borg 6-20 scale); $V A S$, visual analog scale (with terminal anchors of "not at all breathless" and "extremely breathless").

and end-tidal gas tensions for oxygen and carbon dioxide. ${ }^{26}$ The $\dot{V} \mathrm{O}_{2} \theta$ was compared with the lower limit of normal. ${ }^{27}$ Five patients reached a $\dot{V}_{E} \max >85 \%$ of their ventilatory capacity (as measured by maximum voluntary ventilation over 12 seconds) and might thus have been considered to be ventilation limited. For the constant $\dot{W}$ tests, $\dot{V}_{2} \max , f_{c}$ max and $\dot{V}_{E}$ max were higher, with $90 \%$ of $\dot{W} \max$ rather than $80 \%$, suggesting that the $90 \%$ tests were more successful at eliciting a true maximal effort (see Table 2). Ratings of perceived exertion and breathlessness were similar for the incremental and $90 \%$ constant work rate tests. Comparing $80 \%$ and $90 \%$ tests, coefficients of variation were endurance time $16 \%$ versus $17 \%, \dot{V} O_{2} \max 10 \%$ versus $7 \%, \dot{V} C_{2} \max$ $10 \%$ versus $8 \%, f_{c} \max 6 \%$ versus $3 \%$, and $\dot{V}_{E} \max 10 \%$ versus $7 \%$. We concluded from these data that the symptom-limited incremental treadmill protocol was well tolerated in patients with moderate to very severe COPD. Constant $\dot{W}$ tests at $90 \%$ compared with $80 \%$ of $\dot{W}$ max resulted in a narrower range of endurance times and slightly more repeatable physiologic measures. However, the $90 \%$ tests always followed the $80 \%$ tests and so there could have been a sequence effect related to familiarization with the exercise protocol.

\section{EXACTT study}

\section{Subjects}

Five hundred nineteen patients were randomized into the EXACTT study. The demographics and baseline characteristics of these subjects are shown in Table 3. There were 401 men and 118 women with a mean age of $64.6 \pm 8.3$ years. The mean postbronchodilator $\mathrm{FEV}_{1}$ was $44.3 \% \pm 11.9 \%$ of predicted. According to the Global Initiative for Chronic Obstructive Lung Disease (GOLD) criteria, ${ }^{28} 187$ patients had moderate (Stage II) COPD, 261 had severe (Stage III) COPD, and 71 patients had very severe (Stage IV) COPD. The cohort comprised 34.3\% current smokers and $65.7 \%$ former smokers. On average, they had smoked for approximately $51.7 \pm 27.7$ pack years and

Table 3 EXACTT patient demographics and baseline characteristics

\begin{tabular}{|c|c|}
\hline Parameter & Result \\
\hline Men/women, $\mathrm{n}$ & $401 / 118$ \\
\hline Age (years) & $64.6(8.3)$ \\
\hline Body mass index $\left(\mathrm{kg} / \mathrm{m}^{2}\right)$ & $26.4(4.2)$ \\
\hline Current/former smokers & $|78 / 34|$ \\
\hline Smoking history (pack-years) & $51.7(27.7)$ \\
\hline Duration of COPD (years) & $8.8(6.7)$ \\
\hline Prebronchodilator FEV $(\mathrm{L})$ & $1.08(0.40)$ \\
\hline Prebronchodilator FEV (\% predicted) & $38.2(11.2)$ \\
\hline Postbronchodilator FEV, (L) & $1.25(0.42)$ \\
\hline Postbronchodilator FEV (\% predicted) & 44.3 (11.9) \\
\hline $\mathrm{FVC}(\mathrm{L})$ & $2.93(0.72)$ \\
\hline $\mathrm{FEV}_{\mathrm{I}} / \mathrm{FVC}(\%)$ & $50.7(13.5)$ \\
\hline Concurrent LABA, n (\%) & $300(57.8)$ \\
\hline Concurrent ICS, n (\%) & $311(59.9)$ \\
\hline
\end{tabular}

Note: Values are mean (SD) unless otherwise stated.

Abbreviations: COPD, chronic obstructive pulmonary disease; $\mathrm{FEV}_{1}$, forced expiratory volume in one second; FVC, forced vital capacity; LABA, long-acting $\beta$-agonist; ICS, inhaled corticosteroid. 
were known to have COPD for $8.7 \pm 6.6$ years. The number of patients with evaluable data at visits 1,2 , and 3 were 512, 459, and 463 , respectively.

\section{Incremental test}

The mean \pm SD exercise endurance time for the incremental exercise test at visit 1 was $522 \pm 172$ seconds. Individual exercise endurance times ranged from 20 seconds to 890 seconds (see Figure 2). The maximum work rate was $66.3 \pm 33.9$ watts. Values of endurance time and work rate for different stages of COPD severity are shown in Table 4.

\section{Constant work rate tests}

The mean \pm SD and median endurance times for the constant $\dot{W}$ tests were $316 \pm 200$ and 260 seconds, respectively, on visit 2 compared with $341 \pm 184$ and 290 seconds on visit 3 (see Figures 3 and 4). The range of values for visit 2 was 42-1096 seconds and for visit 3 or visit 3r (with work rate adjustment) the range was $42-900$ seconds. The constant work rates for the two visits were $61.0 \pm 32.4$ and $60.4 \pm 32.6$ watts. Values of endurance time and work rate for the different stages of COPD severity are shown in Table 4. Data were available for identical constant $\dot{W}$ tests at visits 2 and 3 in 470 subjects. The target endurance time for the constant work rate tests was 120-900 seconds. If endurance time was $>900$ seconds, the work rate was increased, whereas if endurance time was $<120$ seconds, the work rate was reduced. Increases or reductions in the constant work rate were obtained using speed and grade from either the previous or next stage of the standard protocol. Constant work rate was modified 56 times in 52 subjects, being reduced 32 times in 31 subjects and increased 24 times in 21 subjects. There was good repeatability of endurance times between two identical tests $(n=470)$, with an intraclass correlation coefficient of $0.85(P<0.001)$ for the mean of two tests (see Figure 5).

\section{Locus of symptom limitation}

The reasons for stopping exercise during the constant $\dot{W}$ exercise tests are shown in Table 5. At visit 2, 402 (85.4\%) of the patients stopped exercise due to breathlessness with or without leg fatigue compared with $416(88.3 \%)$ at visit 3 . There was a high degree of consistency $(79.0 \%)$ in the reasons given for stopping exercise, with few patients switching reasons between these two visits.

\section{Safety}

At the time of this analysis, 422 (81\%) of subjects have been retained in the EXACTT trial. Overall, 50 subjects experienced 76 adverse events. Events which occurred in $>1 \%$ of patients were COPD exacerbation (seven patients) and cough (five patients). Two patients experienced serious adverse events, ie, COPD exacerbation and hypotension. The hypotension occurred shortly after exercise testing, but no other serious

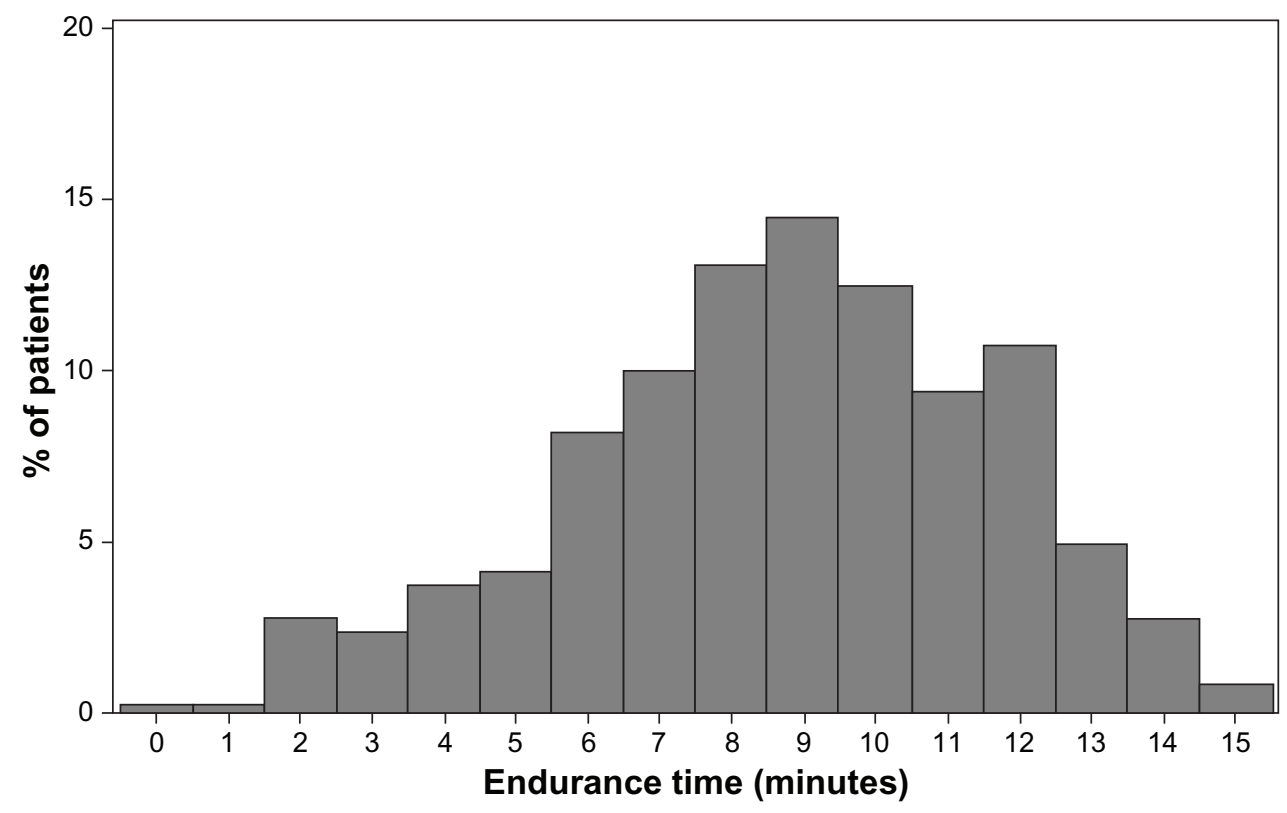

Figure 2 Distribution of patients by endurance time for the incremental exercise tests at visit I $(n=5 \mid 2)$. The mean \pm standard deviation exercise endurance time was $522 \pm 172$ seconds. 
Table 4 Duration and work rate from the EXACTT incremental and constant work rate exercise tests

\begin{tabular}{|c|c|c|c|c|c|c|}
\hline \multirow[t]{2}{*}{ GOLD stage } & \multicolumn{2}{|l|}{ Visit I } & \multicolumn{2}{|l|}{ Visit 2} & \multicolumn{2}{|l|}{ Visit 3} \\
\hline & Time sec & $\dot{W} \max$ watts & Time sec & $\dot{W}$ con watts & Time sec & $\dot{W}$ con watts \\
\hline $\begin{array}{l}\text { Stage II: moderate } \\
(\mathrm{n}=185)\end{array}$ & $557(168)$ & $74(34)$ & 340 (209) & $69(34)$ & 361 (178) & $68(34)$ \\
\hline $\begin{array}{l}\text { Stage III: severe } \\
(\mathrm{n}=260)\end{array}$ & $517(179)$ & $65(34)$ & 305 (197) & $60(32)$ & $321(180)$ & $59(33)$ \\
\hline $\begin{array}{l}\text { Stage IV: very severe } \\
(n=7 I)\end{array}$ & 443 (I28) & $50(22)$ & $295(182)$ & $45(2 I)$ & $362(207)$ & $45(22)$ \\
\hline $\begin{array}{l}\text { All stages } \\
(\mathrm{n}=516)\end{array}$ & $522(172)$ & $66(34)$ & $317(201)$ & $61(33)$ & 341 (184) & $61(33)$ \\
\hline
\end{tabular}

Note: Values are means ( \pm standard deviations).

Abbreviations: Visit I, incremental work rate test; visit 2, constant work rate test at $90 \%$ of maximum work rate from visit I; visit 3, repeat identical constant work rate test; time, endurance time in seconds; $\dot{W}$ max, maximum work rate for the incremental exercise test at visit $I$; $\dot{W}$ con, constant work rate for the exercise tests at visits 2 and 3; GOLD, global initiative for chronic obstructive lung disease.

events were associated with exercise testing. Electrocardiograms were monitored and there were only two reports of premature ventricular contractions and one report of self-limiting ventricular tachycardia. Nonserious advents which occurred on more than one occasion during exercise testing were vertigo (three patients), dizziness or lightheadedness (four patients), and abdominal pain (two patients). There have been no reports of accidental falls or problems with instability.

\section{Discussion}

The purpose of the present study was to develop incremental and constant work rate treadmill exercise testing protocols and then to implement them in the international, multicenter EXACTT study. The primary outcome measure in EXACTT will be endurance time at $90 \%$ of maximum work rate. All patients enrolled were able to complete the incremental and constant work exercise tests, and no subjects were lost to follow-up because of technical difficulties with test administration. Determination of exercise endurance time does not depend upon gas exchange measurements, thus simplifying the test protocols and allowing them to be performed quickly and cost effectively.

The incremental treadmill test was designed based on calculated increases in speed and grade to obtain a linear

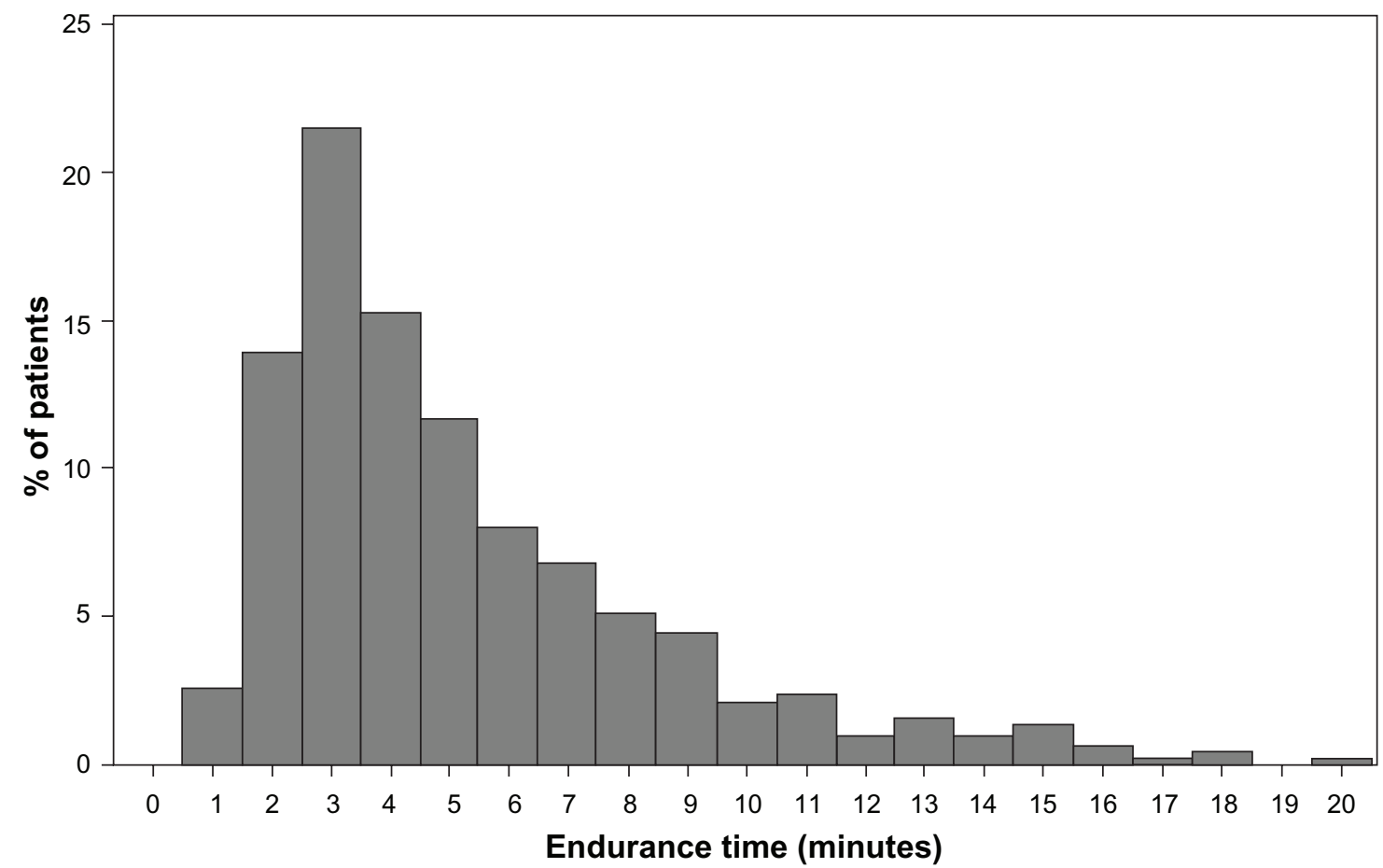

Figure 3 Distribution of endurance time for constant $\dot{W}$ exercise tests at visit $2(n=459)$. The mean \pm standard deviation and median endurance times were $316 \pm 200$ and 260 seconds, respectively. 


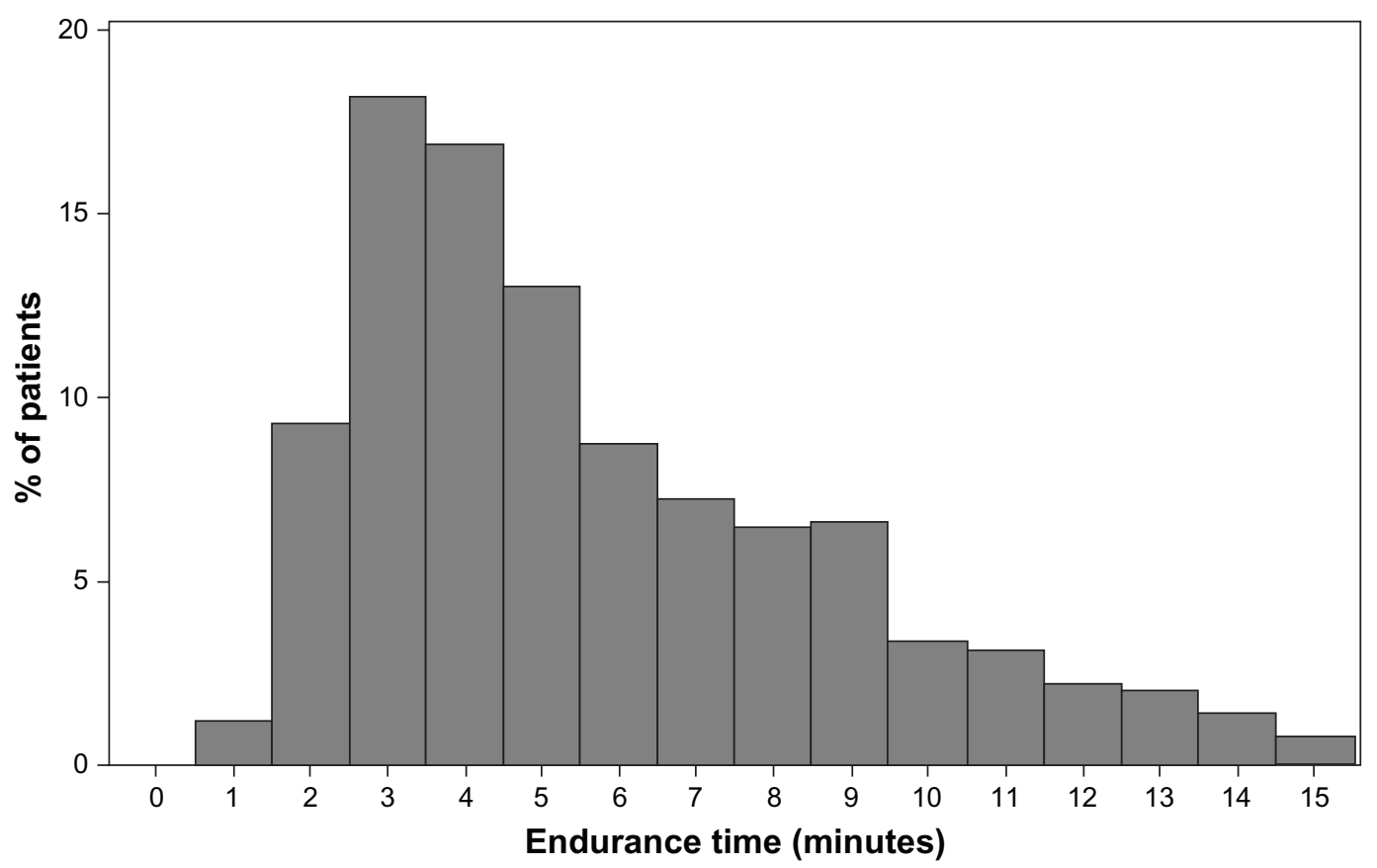

Figure 4 Distribution of endurance time for constant $\dot{W}$ exercise tests at visit $3(n=463)$. The mean \pm standard deviation and median endurance times were $34 \mathrm{I} \pm 184$ and 290 seconds, respectively.

increase in $\dot{V} O_{2}$ suitable for patients with COPD. Plots of $\dot{V} O_{2}$ versus time from the subjects in the pilot study confirm that this objective was achieved. The data also show a mean incremental test time of approximately 10 minutes, with most tests falling within the target range of 8-12 minutes. In the pilot study, subjects attained $80 \%$ of predicted maximum heart rate, and $\dot{\mathrm{V}} \mathrm{O}_{2}$ max was $86 \%$ of that predicted for sedentary individuals. ${ }^{16}$ Furthermore, $7 / 12$ subjects had evidence of a plateau in $\dot{V} O_{2}$ despite further increase in $\dot{W}$, suggesting a true maximal test. Work efficiency was, on average, similar

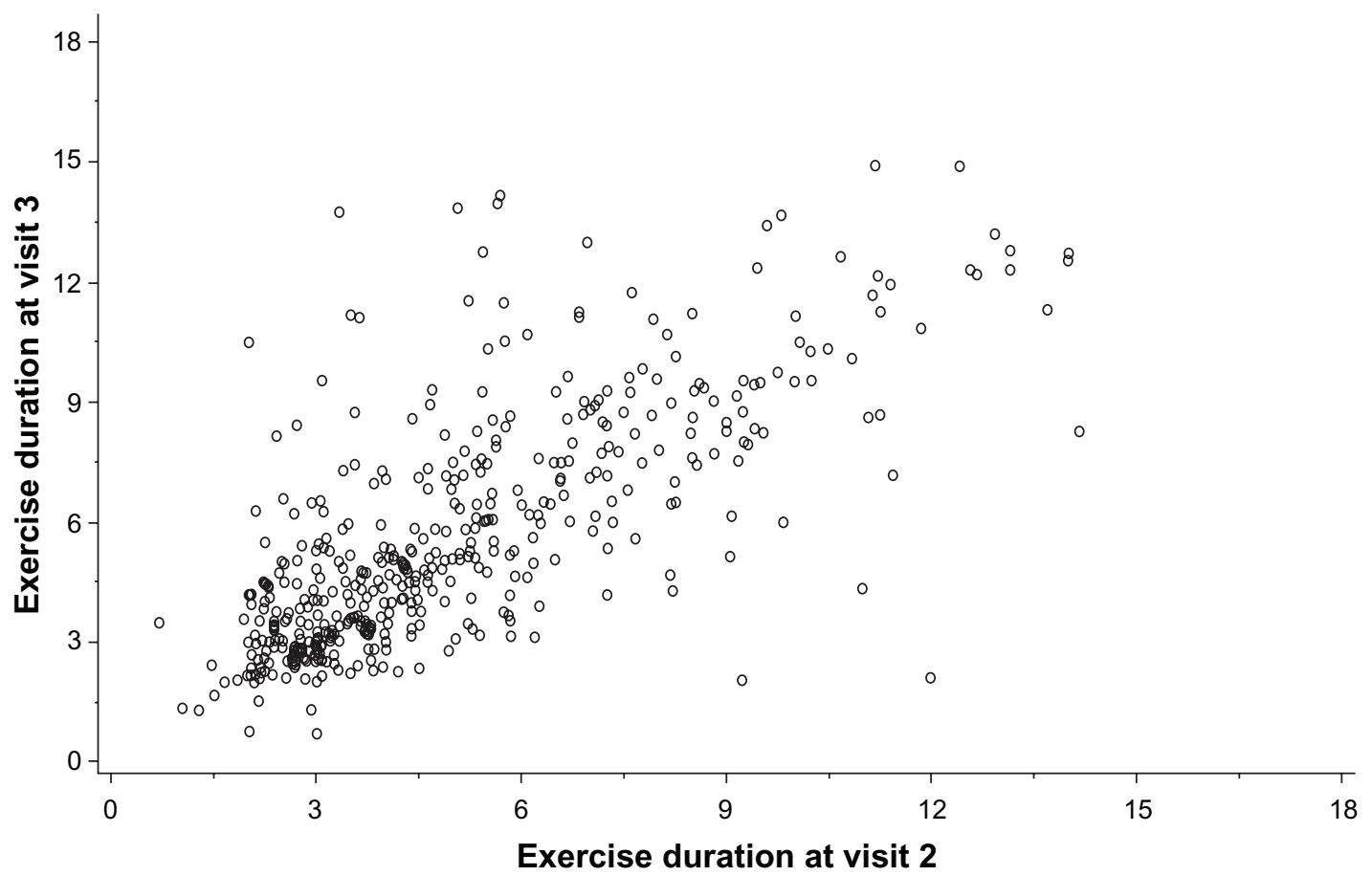

Figure 5 Comparison of endurance times for constant $\dot{W}$ exercise tests at visits 2 and 3 . The means of the two tests had an intraclass correlation coefficient of 0.85 ( $P<0.00 \mathrm{I})$. 
Table 5 Reason for stopping the constant work rate exercise test at randomization in EXACTT (visit 3)

\begin{tabular}{llll}
\hline GOLD stage & $\begin{array}{l}\text { Reason for } \\
\text { stopping }\end{array}$ & Number & $\begin{array}{l}\text { Percentage } \\
\text { within stage }\end{array}$ \\
\hline $\begin{array}{llll}\text { Stage II: moderate } \\
(\mathrm{n}=\text { I87) }\end{array}$ & $\begin{array}{l}\text { Breathing } \\
\text { discomfort }\end{array}$ & 107 & 57.2 \\
& Leg discomfort & 23 & 12.3 \\
& Both & 56 & 29.9 \\
& Neither & 1 & 0.5 \\
& Any breathing & 163 & 87.1 \\
Stage III: severe & Breathing & 170 & 65.1 \\
$(\mathrm{n}=26 \mathrm{I})$ & discomfort & & \\
& Leg discomfort & 26 & 10.0 \\
& Both & 61 & 23.4 \\
& Neither & 4 & 1.5 \\
& Any breathing & 23 I & 88.5 \\
Stage IV: very severe & Breathing & 45 & 63.4 \\
$(\mathrm{n}=7 \mathrm{I})$ & discomfort & & \\
& Leg discomfort & 6 & 0.1 \\
& Both & 19 & 26.8 \\
& Neither & 1 & 1.4 \\
& Any breathing & 64 & 90.2 \\
& Breathing & 322 & 62.0 \\
All stages & discomfort & & \\
$(\mathrm{n}=5$ |9) & Leg discomfort & 55 & 10.6 \\
& Both & 136 & 26.2 \\
& Neither & 6 & 1.1 \\
& Any breathing & 458 & 88.2 \\
\hline
\end{tabular}

Notes: Values are absolute numbers in each category followed by the percentage of all patients with the same stage of chronic obstructive pulmonary disease severity. "Any breathing" refers to the combined number of patients in the "breathing discomfort" and "both" categories.

to what would be expected with incremental cycle ergometry. ${ }^{24}$ Only $5 / 12$ subjects exhibited ventilatory limitation $\left(\dot{V}_{E} \max >85 \%\right.$ of maximum voluntary ventilation measured directly over 12 seconds prior to exercise testing). This is a typical finding in COPD patients where there can be other reasons for exercise limitation. In summary, this incremental $\dot{W}$ protocol, specific for patients with COPD, is novel, feasible, and well tolerated by patients with COPD ranging from moderate to very severe by GOLD criteria. ${ }^{28}$

The EXACTT study will evaluate the long-term effect of tiotropium on exercise tolerance in patients with COPD over two years. The rationale for this study is based on the results of several short-term clinical trials in patients with COPD demonstrating improvement with tiotropium $18 \mu \mathrm{g}$ once daily on exercise duration using different types of exercise protocol. ${ }^{6,714,29}$ For example, two six-week protocols evaluated constant work cycle ergometry, ${ }^{6,7}$ one 12-week study examined the incremental shuttle walk test, ${ }^{29}$ and a fourth study examined constant speed treadmill testing as an outcome in patients receiving tiotropium or matching placebo in combination with pulmonary rehabilitation. ${ }^{14}$
Commonly used exercise tests have several limitations. Standard protocols for cardiopulmonary exercise testing, such as the Bruce protocol, are generally too demanding for COPD patients and were designed for other purposes. ${ }^{30}$ The six-minute walking test has gained acceptance in a variety of chronic pulmonary diseases, but is subject to preset behaviors and has been shown to be administered inconsistently. ${ }^{3}$ This type of functional exercise test exhibits large withinsubject variability from day to day, and therefore requires considerable attention to standardization. ${ }^{2}$ For example, it has been shown to be nonresponsive to bronchodilator therapy despite improvement in $\mathrm{FEV}_{1}$, and also in shuttle walking performance. ${ }^{31}$ The endurance shuttle walk test may be more responsive than the six-minute walking test, ${ }^{31}$ but it could also have limitations given the need to move back and forth on a corridor and to perform sharp turns which might cause instability. Revill et $\mathrm{al}^{8}$ noted the energy expenditure will likely be higher in the shuttle walk compared with a treadmill test due to the turning of corners. Furthermore, a 20-minute ceiling effect has been recognized with the shuttle test which could pose problems in the evaluation of higher functioning individuals. ${ }^{32} \mathrm{~A}$ potential benefit of constant work treadmill testing is that there may not be a ceiling effect in higher functioning subjects.

Constant work cycle ergometry has an accepted, standardized methodology and has proven to be responsive to pharmacologic interventions. ${ }^{6,7,33}$ However, cycling is not usually a habitual activity for COPD patients, and involves different muscle recruitment than walking. Cycle ergometry may mask true treatment effects that improve ventilatory mechanics due to cessation of work from leg fatigue. Pepin et $\mathrm{al}^{11}$ evaluated changes in endurance time during cycling and walking (endurance shuttle test) in 17 patients with COPD with placebo and ipratropium. Endurance time increased by a mean of 51 seconds with cycling and by a mean of 164 seconds with walking. Quadriceps twitch force was reduced with cycling, but not with walking and, furthermore, there was a shift in the locus of symptom limitation towards dyspnea rather than leg fatigue with the walking test. These observations suggest that walking endurance tests minimize the contribution of leg fatigue to exercise limitation, and should therefore be more sensitive for detecting improvements in dyspnea related to alterations in ventilatory mechanics. These issues highlight the need for an endurance walking test with a standardized protocol that is applicable in COPD patients. Acceptance of a constant work rate endurance test for the evaluation of pharmacologic interventions will certainly require wider experience of such 
testing and comparison with functional exercise tests, such as the six-minute walking test.

An optimal test duration of 8-12 minutes is often quoted for maximal incremental exercise testing. ${ }^{15}$ According to the power-duration curve, there is a nonlinear relationship between intensity of a task and the duration for which the task can be performed. Thus, a too low intensity results in prolonged exercise duration, masking ventilatory limitation and limiting ability to detect true intervention effects. By contrast, a too high intensity results in insufficient data for interpretation. A recent study of COPD patients (Stages III and IV) performing incremental cycle ergometry suggests an optimal test duration of approximately 5-9 minutes, as compared with 8-12 minutes for normal subjects. ${ }^{34}$

The duration of the second treadmill constant $\dot{W}$ tests at $90 \%$ of $\dot{W} \max$ on visit 3 of the EXACTT study was 340 seconds. Notably, there were several tests of prolonged duration (one being almost 20 minutes). An obvious reason for such prolonged endurance is that the initial incremental test underestimated true $\dot{W} \max$, and thus $90 \%$ of this value represented a relatively low intensity. Such prolonged tests need to be avoided in the setting of clinical trials where endurance time is an important clinical endpoint. For this reason, for 21 subjects in EXACTT whose endurance time exceeded 15 minutes, we adjusted the constant $\dot{W}$ and repeated the test. For the 470 subjects performing identical constant $\dot{W}$ tests at visits 2 and 3, there was good repeatability of endurance times between the two tests (intraclass correlation 0.85). We considered this degree of variation to be an acceptable baseline for the EXACTT clinical trial.

This study has several limitations. Firstly, it is recognized that the feasibility study included a low number of patients. However, it appeared that patients tolerated the protocol and it was feasible to institute. Secondly, in the EXACTT study, we excluded subjects who were obese, but recognize that a significant proportion of COPD patients do have a body mass index $>30$. The EXACTT protocol might not be so easily tolerated in heavier subjects, and this will have to be evaluated separately. Thirdly, we deliberately chose to increment the work rate slowly at the beginning of the incremental exercise tests. This was to improve subject comfort during testing. We acknowledge, however, that this might have led to an underestimation of peak power, especially in subjects with lower functional capacity. Had this phenomenon occurred, our selection of $90 \%$ of maximum work rate for the endurance tests would be even more appropriate. Another recognized limitation of the study is the exclusion of patients with an $\mathrm{FEV}_{1}>65 \%$. However, we believe that our subject population is representative of the type of patient seen in clinical practice. Finally, regarding differences in exercise duration between repeated visits, the possibility of a learning effect cannot be excluded.

We have sought to minimize subjective perception of leg fatigue as the factor limiting exercise so as to have a stimulus that would maximize the potential to demonstrate true improvements in ventilatory mechanics. A large international trial involving constant work cycle ergometry suggested that leg fatigue (either alone or in combination with breathlessness) was responsible for $53 \%-66 \%$ of patients reason for stopping exercise. ${ }^{7}$ The treadmill protocol we developed resulted in $88 \%$ of subjects reporting breathing discomfort alone or in conjunction with leg fatigue as the locus of symptom limitation. This feature renders the EXACTT protocol particularly suitable for eliciting potential improvements in exercise capacity where there is limitation by dyspnea.

\section{Conclusions}

In summary, the maximal incremental treadmill exercise test protocols we have developed were well tolerated by patients with moderate to very severe COPD. We believe that treadmill testing is more representative of the physiologic limitations of the COPD patient than cycle ergometry, particularly given that our data show that breathlessness is more likely to be the limiting factor in treadmill exercise tests. Additionally, the test protocols we have developed may be performed easily in a variety of clinical settings. They are being further evaluated as part of a multinational clinical trial.

\section{Disclosure}

CC currently holds research contracts with Boehringer Ingelheim Pharmaceuticals and Spiration, and research grants from The Alpha-1 Foundation, Breathe California, and The March of Dimes Research Foundation. He serves on scientific advisory boards for VIASYS Clinical Services, ROX Medical, Boehringer Ingelheim Pharmaceuticals, and Pfizer. He has received honoraria for speaking, along with reimbursement of expenses, from AstraZeneca, Boehringer Ingelheim Pharmaceuticals, and Pfizer. MA is a member of staff of the UCLA Exercise Physiology Research Laboratory and has no conflicts of interest to disclose. DL and SK are full time employees of Boehringer Ingelheim Pharmaceuticals, Inc.

\section{References}

1. Cooper CB. Exercise in chronic pulmonary disease: Limitations and rehabilitation. Med Sci Sports Exerc. 2001;33:S643-S646.

2. ATS statement: Guidelines for the six-minute walk test. Am J Respir Crit Care Med. 2002;166:111-117. 
3. Elpern EH, Stevens D, Kesten S. Variability in performance of timed walk tests in pulmonary rehabilitation programs. Chest. 2000;118: 98-105.

4. Mahler DA, Donohue JF, Barbee RA, et al. Efficacy of salmeterol xinafoate in the treatment of COPD. Chest. 1999;115:957-965.

5. Oga T, Nishimura K, Tsukino M, et al. The effects of oxitropium bromide on exercise performance in patients with stable chronic obstructive pulmonary disease. A comparison of three different exercise tests. Am J Respir Crit Care Med. 2000;161:1897-1901.

6. O’Donnell DE, Fluge T, Gerken F, et al. Effects of tiotropium on lung hyperinflation, dyspnoea and exercise tolerance in COPD. Eur Respir J. 2004;23:832-840.

7. Maltais F, Hamilton A, Marciniuk D, et al. Improvements in symptomlimited exercise performance over $8 \mathrm{~h}$ with once-daily tiotropium in patients with COPD. Chest. 2005;128:1168-1178.

8. Revill SM, Morgan MD, Singh SJ, et al. The endurance shuttle walk: A new field test for the assessment of endurance capacity in chronic obstructive pulmonary disease. Thorax. 1999;54:213-222.

9. Man WD, Soliman MG, Gearing J, et al. Symptoms and quadriceps fatigability after walking and cycling in chronic obstructive pulmonary disease. Am J Respir Crit Care Med. 2003;168:562-567.

10. Saey D, Debigare R, LeBlanc P, et al. Contractile leg fatigue after cycle exercise: A factor limiting exercise in patients with chronic obstructive pulmonary disease. Am J Respir Crit Care Med. 2003;168:425-430.

11. Pepin V, Saey D, Whittom F, et al. Walking versus cycling: Sensitivity to bronchodilation in chronic obstructive pulmonary disease. $\mathrm{Am} \mathrm{J}$ Respir Crit Care Med. 2005;172:1517-1522.

12. Kaminsky LA, Whaley MH. Evaluation of a new standardized ramp protocol: The BSU/Bruce Ramp protocol. J Cardiopulm Rehabil. 1998; 18:438-444

13. Hsia D, Casaburi R, Pradhan A, et al. Physiological responses to linear treadmill and cycle ergometer exercise in COPD. Eur Respir J. 2009; 34:605-615.

14. Casaburi R, Kukafka D, Cooper CB, et al. Improvement in exercise tolerance with the combination of tiotropium and pulmonary rehabilitation in patients with COPD. Chest. 2005;127:809-817.

15. Buchfuhrer MJ, Hansen JE, Robinson TE, et al. Optimizing the exercise protocol for cardiopulmonary assessment. J Appl Physiol. 1983;55: 1558-1564.

16. Cooper CB, Storer TW. Exercise Testing and Interpretation: A Practical Approach. New York, NY: Cambridge University Press; 2001.

17. Porszasz J, Casaburi R, Somfay A, et al. A treadmill ramp protocol using simultaneous changes in speed and grade. Med Sci Sports Exerc. 2003;35:1596-1603.

18. Borg G. Perceived exertion as an indicator of somatic stress. Scand J Rehabil Med. 1970;2:92-98.
19. American Thoracic Society. Standardization of Spirometry, 1994 Update. American Thoracic Society. Am J Respir Crit Care Med. 1995; 152:1107-1136.

20. Miller MR, Hankinson J, Brusasco V, et al. Standardisation of spirometry. Eur Respir J. 2005;26:319-338.

21. Hankinson JL, Odencrantz JR, Fedan KB. Spirometric reference values from a sample of the general U.S. population. Am J Respir Crit Care Med. 1999;159:179-187.

22. Petrie A, Sabin C. Medical Statistics at a Glance. 2nd ed. Malden, MA: Blackwell; 2005

23. Shrout PE, Fleiss JL. Intraclass correlations: Uses in assessing rater reliability. Psychol Bull. 1979;86:420-428.

24. Whipp BJ, Davis JA, Torres F, et al. A test to determine parameters of aerobic function during exercise. J Appl Physiol. 1981;50:217-221.

25. Beaver WL, Wasserman K, Whipp BJ. A new method for detecting anaerobic threshold by gas exchange. J Appl Physiol. 1986;60: 2020-2027.

26. Marques-Magallanes JA, Smooke S, Sehdeva PS, et al. Reliability of gas exchange threshold detection (VO2H) comparing blinded investigators with computer algorithms. Am J Respir Crit Care Med. 1997;155:A170.

27. Davis JA, Storer TW, Caiozzo VJ. Prediction of normal values for lactate threshold estimated by gas exchange in men and women. Eur J Appl Physiol Occup Physiol. 1997;76:157-164.

28. Global strategy for the diagnosis, management, and prevention of chronic obstructive pulmonary disease. NHLBI/WHO workshop summary, updated 2007. London: Global Initiative for Chronic Obstructive Lung Disease (GOLD), Dec 2007. Available from: http://www.ginasthma.com/ download.asp?intid=309 Accessed Jul 24, 2010.

29. Verkindre C, Bart F, Aguilaniu B, et al. The effect of tiotropium on hyperinflation and exercise capacity in chronic obstructive pulmonary disease. Respiration. 2006;73:420-427.

30. Bruce RA. Exercise testing of patients with coronary heart disease. Principles and normal standards for evaluation. Ann Clin Res. 1971;3: 323-332.

31. Pepin V, Brodeur J, Lacasse Y, et al. Six-minute walking versus shuttle walking: Responsiveness to bronchodilation in chronic obstructive pulmonary disease. Thorax. 2007;62:291-298.

32. Brooks D, Solway S. Should the endurance shuttle walk test replace the six-minute walk test in individuals with chronic obstructive pulmonary disease? Chron Respir Dis. 2006;3:1-2.

33. O'Donnell DE, Voduc N, Fitzpatrick M, et al. Effect of salmeterol on the ventilatory response to exercise in chronic obstructive pulmonary disease. Eur Respir J. 2004;24:86-94.

34. Benzo RP, Paramesh S, Patel SA, et al. Optimal protocol selection for cardiopulmonary exercise testing in severe COPD. Chest. 2007;132: $1500-1505$.
International Journal of COPD

\section{Publish your work in this journal}

The International Journal of COPD is an international, peer-reviewed journal of therapeutics and pharmacology focusing on concise rapid reporting of clinical studies and reviews in COPD. Special focus is given to the pathophysiological processes underlying the disease, intervention programs, patient focused education, and self management protocols.

\section{Dovepress}

This journal is indexed on PubMed Central, MedLine and CAS. The manuscript management system is completely online and includes a very quick and fair peer-review system, which is all easy to use. Visi http://www.dovepress.com/testimonials.php to read real quotes from published authors. 